\title{
Características de los planes de estudio en el desarrollo de investigación científica en la Odontología peruana
}

\section{Characteristics of study plans on the development of research in Peruvian Dentistry}

\begin{abstract}
Resumen
Objetivo. Describir las características de los planes de estudio de las facultades peruanas de Odontología respecto a la implementación de cursos relacionados a la investigación científica. Métodos. Estudio descriptivo que recolectó los planes de estudio de $42 \mathrm{fa}$ cultades de Odontología vigentes en el Perú hasta el 2019. En cada plan de estudio se recolectó los datos de: tipo de universidad, cantidad de años de duración de la profesión, cantidad de asignaturas relacionadas a investigación, semestre de inicio de los cursos de investigación y cantidad de cursos relacionados a la tesis universitaria. Se realizaron comparaciones de promedios y errores estándar según tipo de universidad (públicas/privadas). Resultados. El promedio de cursos relacionados a la investigación fue de 4,5 \pm 1,7; siendo la mediana de 4, con un valor mínimo de 1 y un valor máximo de 9 . Las universidades públicas presentaron una mayor cantidad de asignaturas relacionadas a la investigación $(5,1 \pm 0,6)$ a diferencia de las universidades privadas que presentaron un promedio de $1,3 \pm 0,2$. Dos universidades privadas presentaron mayor cantidad de cursos con el término tesis en sus planes de estudio. Conclusiones. Las universidades públicas presentaron una mayor cantidad de asignaturas relacionadas a la investigación. Las universidades privadas en promedio tuvieron mayor cantidad de cursos relacionados a tesis.
\end{abstract}

Palabras clave: Currículum; Odontología; Investigación; Educación basada en competencias (fuente: DeCS BIREME).

\begin{abstract}
Objective. To describe the characteristics of study plans of Peruvian Dentistry faculties regarding the implementation of courses related to scientific research. Methods. A descriptive study that gathered the curricula of the 42 dentistry faculties in Peru until 2019. In each study plan, the following data were collected: type of university, number of years of duration of the profession, number of subjects related to research, semester of the beginning of the research courses and number of courses related to the university thesis. Comparisons of averages and standard errors were made according to type of university (state / private). Results. The average of courses related to the research was
\end{abstract}

\section{Artículo Original}

Olenka Valenzuela-Torres ${ }^{1, a}$, Stefany Maza-Cano ${ }^{1, a}$, Valeria Sihuay-Torres ${ }^{2, a}$, Yuri Castro-Rodríguez ${ }^{3, b}$

${ }^{1}$ Universidad Nacional Mayor de San Marcos, Sociedad Científica de Estudiantes de Odontología. Lima, Perú.

${ }^{2}$ Universidad Nacional Federico Villarreal, Sociedad Científica de Estudiantes de Odontología. Lima, Perú. ${ }^{3}$ Universidad Privada Juan Pablo II, Facultad de Ciencias de la Salud. Lima, Perú.

a Estudiante del pregrado.

${ }^{\mathrm{b}}$ Magíster en Educación.

\section{Correspondencia:}

Olenka Valenzuela Torres

Correo electrónico: olenkayvt18@hotmail.com San Remo 2. Mz.G lote 53. San Martín de Porres. Lima, Perú.

\section{Coautores:}

Stefany Maza Cano

stefany.maza@unmsm.edu.pe

Valeria Sihuay Torres

Vale.Sihuay@gmail.com

Yuri Castro Rodríguez

yuricastro_16@hotmail.com

\section{Editora:}

Dahiana Alfaro Carballido

Universidad de San Martín de Porres, Perú.

Conflicto de intereses: los autores declaran no tener conflictos de interés.

Fuente de financiamiento: autofinanciado.

Recibido: 05/02/19

Aceptado: 05/03/19

Publicado: 05/06/19 
$4.5 \pm 1.7$; being the median of 4 with a minimum value of 1 and a maximum value of 9 . Public universities presented a greater number of subjects related to research $(5.1 \pm 0.6)$ unlike private universities that presented an average of $1.3 \pm 0.2$. Two private universities presented more courses with term thesis in their study plans. Conclusions. The state universities presented a greater number of subjects related to research. On average, private universities had a greater number of thesis-related courses.

Keywords: Curriculum; Dentistry; Research; Competency-based education (source: MeSH NLM).

\section{Introducción}

En la educación médica la investigación científica se reconoce como uno de los pilares para su desarrollo. Esto se debe a que los médicos-investigadores son capaces de generar nuevos conocimientos en pro de la mejora de la salud de los pacientes además porque la investigación permite desarrollar habilidades estudiantiles como el pensamiento crítico, la creatividad, el sentido ético, el razonamiento cuantitativo, la orientación al logro y el aprendizaje autónomo ${ }^{1,2}$.

Debido a la importancia de la investigación científica es necesario que el plan de estudio de una facultad involucre una serie de contenidos que confieran a los estudiantes las habilidades necesarias para realizar trabajos de investigación, desde su formulación hasta su culminación incluyendo el proceso de publicación en revistas científicas indizadas.

Los planes de estudio universitarios deben actualizarse constantemente para lograr el desarrollo de una carrera profesional; lo cual implica evaluar el plan, modificar al vigente y otorgarle validez a la carrera que respalda. La evaluación de un plan de estudios descubre qué características deben ser actualizadas, las debilidades, fortalezas y fallas que sean necesarias para estar acorde al avance científico-tecnológico que la sociedad y comunidad soli$\operatorname{citan}^{3}$. De esta forma, con el avance tecnológico y científico se requiere que los planes de estudio se adecúen a las nuevas estrategias de enseñanza-aprendizaje que permitan garantizar al estudiante un perfil amplio, que conozca y pueda emplear en su quehacer profesional el método científico y, de forma creadora pueda resolver los problemas que se le planteen durante su futura vida profesional ${ }^{4}$.

En los estudios relacionados a las ciencias de la salud, incluir actividades investigativas dentro de los planes de estudio debe permitir al estudiante desarrollar una actitud científica, lo cual favorecerá el desarrollo de nuevos intereses cognitivos y la motivación por seguir una carrera que es necesaria para el avance de una comunidad ${ }^{4}$. Actualmente, en el Perú se reporta una baja producción científica estudiantil ${ }^{5-8}$, señalando entre las principales limitaciones para realizar y publicar trabajos de investigación la falta de apoyo docente ${ }^{9} \mathrm{y}$ las deficiencias que tienen los estudiantes en temas de metodología de la investigación y publicación ${ }^{10,11}$. Estas deficiencias en parte pueden deberse a que un plan de estudios podría adolecer de cursos como: redacción científica, bioestadística o divulgación científica; considerados como ele- mentos clave para que produzca conocimiento a través de artículos científicos.

Los currículos universitarios deben modificarse cada cierto tiempo debido al avance del conocimiento, tecnologías y necesidades de las poblaciones. En el campo de la Odontología no es diferente esta necesidad y los planes de estudio requieren adaptarse a las necesidades que la actual sociedad del conocimiento requiere. Parte fundamental de tal adaptación es la dedicación a la investigación y producción científica (PC) estudiantil. De esta forma en el presente estudio se describen las características de los planes de estudio de las facultades peruanas de Odontología respecto a la implementación de cursos relacionados a la investigación científica.

\section{Métodos}

Se realizó un estudio observacional, prospectivo, descriptivo y transversal. La población estudiada estuvo conformada por las universidades del Perú que presentaron facultades o escuelas de Odontología y/o Estomatología. La unidad de estudio fueron los planes de estudio de cada facultad seleccionada.

El listado de las universidades que ofertan la profesión de Odontología/Estomatología fue obtenido a partir del portal del Colegio Odontológico del Perú ${ }^{12}$. Acorde a esto se identificó a 42 facultades. En cada portal web se buscó el currículo de pregrado o en su defecto el plan de estudios del pregrado.

En los casos donde no se encontró el plan de estudios ya sea en su formato PDF, HTML u online se procedió a enviar un correo electrónico a las escuelas académicas para solicitar tal documento.

Localizado los planes de estudio se procedió a analizar las siguientes variables: tipo de universidad (nacional y particular), denominación de la profesión (Odontología, Estomatología u otro), cantidad de años de duración de la profesión (considerado desde el ingreso hasta el fin del internado hospitalario), régimen de estudios (anual, semestral o mixto), cantidad de asignaturas, cantidad de asignaturas relacionadas a investigación (se contabilizaron los cursos relacionados a: metodología del trabajo universitario, metodología de la investigación, estadística, bioestadística, redacción científica, tesis, seminario de tesis, epidemiología, proyectos, análisis de datos, análisis de artículos y sus similares), semestre de inicio de los cursos de investigación y cantidad de cursos relacionados a la tesis universitaria (se contabilizó a los cursos que en su denominación tuvieron el término de "tesis"). 
Los datos fueron vaciados en una hoja de cálculo Excel para luego ser analizados en el software SPSS v23.0. Se realizó estadística descriptiva a través de frecuencias relativas y absolutas, así como medidas de tendencia central y dispersión. No fue necesario el uso de la estadística inferencial por haberse estudiado a casi la totalidad de la población de facultades de Odontología del Perú. El presente estudio no requirió de la aprobación de un Comité de Ética puesto que utilizó información de libre acceso en la red.

\section{Resultados}

De las 42 facultades de Odontología registradas en el Colegio Odontológico del Perú al 2019, tres no presentaron sus planes de estudio en sus páginas web y no se tuvo respuesta al requerir los planes vía correo electrónico.

De las 39 facultades analizadas, 10 (25,6\%) fueron públicas y $29(74,4 \%)$ privadas. $23(59 \%)$ facultades tuvieron la denominación de "Odontología" y 16 (41\%) la denominación de "Estomatología" (Tabla).

En promedio la cantidad de asignaturas que se imparten es de 63,7 $\pm 10,9$ teniendo una mediana de 65 con un valor mínimo de 40 y un valor máximo de 92; en promedio las universidades públicas presentaron una mayor cantidad de asignaturas $(66,7 \pm 3,3)$ (Figura 1A).

El promedio de cursos relacionados a la investigación fue de 4,5 $\pm 1,7$; siendo la mediana de 4 con un valor mínimo de 1 y un valor máximo de 9 . Las universidades públicas presentaron una mayor cantidad de asignaturas relacionadas a la investigación $(5,1 \pm 0,6)$ (Figura 1B).

Dos universidades privadas fueron las que más cursos con el término de "tesis" presentaron en sus planes de estudio (Tabla). Las universidades privadas en promedio tuvieron mayor cantidad de cursos relacionados a tesis $(1,3 \pm 0,2)$ (Figura 2A).

La cantidad de cursos clínicos en promedio fue de 20,9 $\pm 1,2$ siendo mayor en las universidades públicas $(21,2$ $\pm 2,9$ ) (Figura 2B).

\section{Discusión}

En las ciencias de la salud hoy se presenta un interés por promover la investigación y producción científica entre los estudiantes universitarios ${ }^{7,13}$; sin embargo, algunos estudiantes indican que no reciben el adecuado apoyo docente y carecen de habilidades en metodología de la investigación y redacción científica ${ }^{6}$.

Resulta clave analizar si los cursos en los planes de estudio son los apropiados y más aún si estos logran desarrollar habilidades investigativas que permitan a un estudiante planificar, ejecutar y difundir sus estudios. Habilidades que se pueden lograr si los docentes dictantes de los cursos relacionados a investigación científica presentan la experiencia y habilidades suficientes en investigación y producción de conocimiento ${ }^{14}$.

La Odontología es una profesión de las ciencias de la salud y la investigación debe ser un pilar en el desarrollo

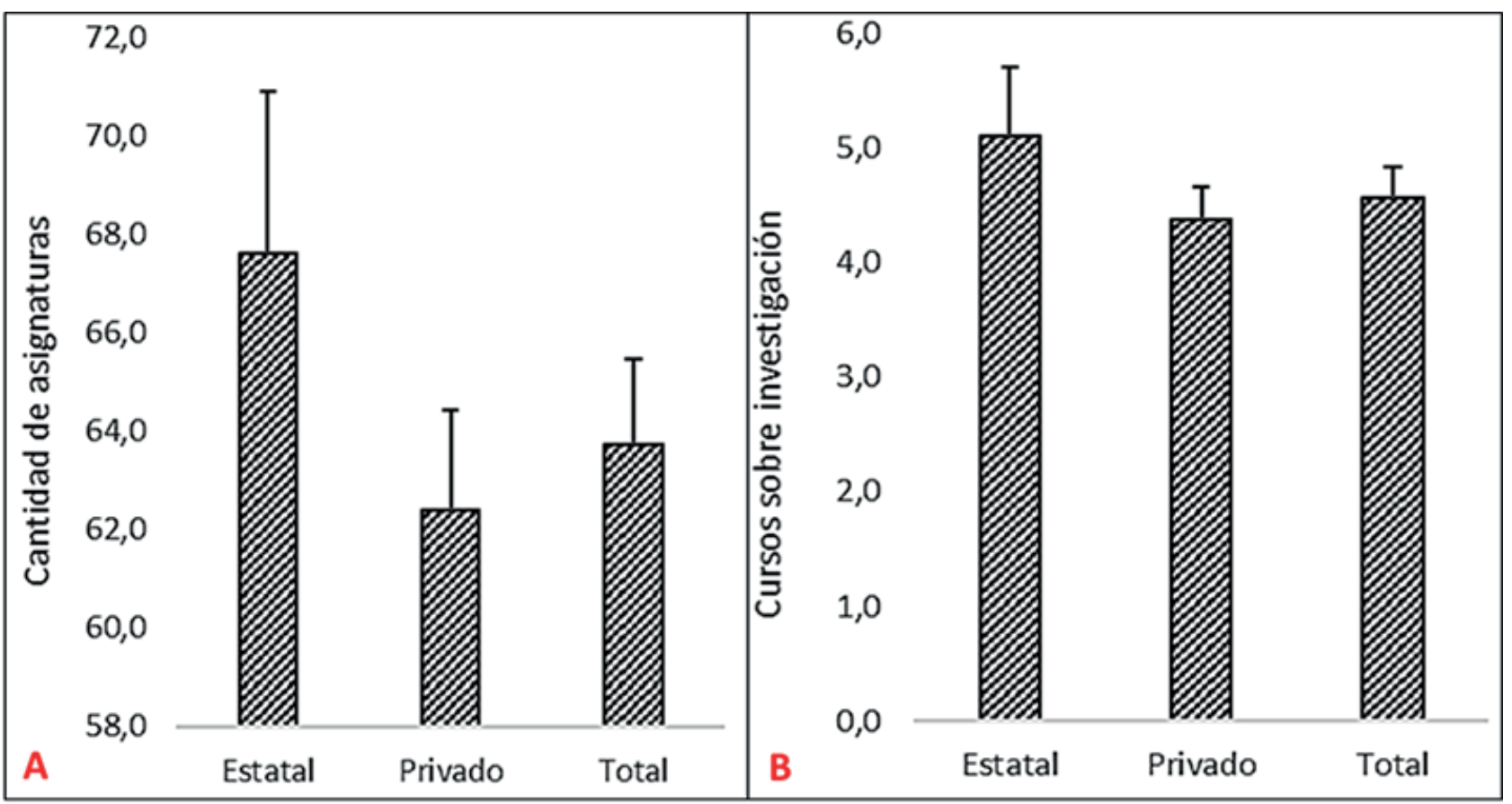

Figura 1. A. Promedio de asignaturas de los planes de estudio por tipo de universidad. B. Promedio de cursos relacionados a investigación científica por tipo de universidad 
Tabla. Cursos relacionados a la investigación y tesis de las universidades del Perú que presentan la carrera profesional de Odontología/Estomatología

\begin{tabular}{|c|c|c|c|c|c|c|c|}
\hline $\mathbf{N}^{\circ}$ & Universidad & Tipo & $\begin{array}{l}\text { Duración } \\
\text { de los } \\
\text { estudios* }\end{array}$ & $\begin{array}{l}\text { Cantidad de } \\
\text { asignaturas }\end{array}$ & $\begin{array}{l}\text { Asignaturas } \\
\text { relacionadas a } \\
\text { investigación }\end{array}$ & $\begin{array}{l}\text { Inicio } \\
\text { de los } \\
\text { cursos }^{\dagger}\end{array}$ & $\begin{array}{l}\text { Cursos con } \\
\text { el término } \\
\text { de tesis }\end{array}$ \\
\hline 1 & Universidad Nacional Mayor de San Marcos & Pública & 6 & 70 & 8 & 4 & 2 \\
\hline 2 & Universidad Nacional Federico Villarreal & Pública & 6 & 67 & 6 & 2 & 0 \\
\hline 3 & Universidad Nacional Toribio Rodríguez de Mendoza & Pública & 6 & 59 & 3 & 2 & 2 \\
\hline 4 & Universidad Nacional Daniel Alcides Carrión & Pública & 6 & 75 & 8 & 2 & 0 \\
\hline 5 & Universidad Nacional Hermilio Valdizán & Pública & 5 & 62 & 5 & 1 & 2 \\
\hline 6 & Universidad Nacional de la Amazonía Peruana & Pública & 6 & 70 & 3 & 2 & 2 \\
\hline 7 & Universidad Nacional del Altiplano & Pública & 5 & 66 & 6 & 1 & 0 \\
\hline 8 & Universidad Nacional Jorge Basadre Grohmann & Pública & 6 & 81 & 4 & 2 & 1 \\
\hline 9 & Universidad Nacional de Trujillo & Pública & 6 & 46 & 3 & 2 & 0 \\
\hline 10 & Universidad Privada Antenor Orrego & Privada & 5 & 80 & 5 & 5 & 2 \\
\hline 11 & Universidad Peruana Cayetano Heredia & Privada & 5 & 71 & 7 & 1 & 0 \\
\hline 12 & Universidad de San Martín de Porres & Privada & 5 & 55 & 5 & 6 & 2 \\
\hline 13 & Universidad Inca Garcilaso de la Vega & Privada & 6 & 67 & 6 & 2 & 0 \\
\hline 14 & Universidad Alas Peruanas & Privada & 5 & 57 & 4 & 1 & 1 \\
\hline 15 & Universidad Científica del Sur & Privada & 5 & 57 & 4 & 3 & 2 \\
\hline 16 & Universidad Privada San Juan Bautista & Privada & 5 & 60 & 4 & 3 & 2 \\
\hline 17 & Universidad Norbert Wiener & Privada & 5 & 56 & 3 & 3 & 1 \\
\hline 18 & Universidad Peruana de Ciencias Aplicadas & Privada & 5 & 48 & 5 & 1 & 2 \\
\hline 19 & Universidad Peruana los Andes & Privada & 5 & 79 & 6 & 1 & 0 \\
\hline 20 & Universidad Privada Telesup & Privada & 5 & 51 & 3 & 2 & 1 \\
\hline 21 & Universidad Privada Juan Pablo II & Privada & 5 & 59 & 6 & 3 & 3 \\
\hline 22 & Universidad Tecnológica de los Andes & Privada & 5 & 40 & 1 & 8 & 1 \\
\hline 23 & Universidad Católica de Santa María & Privada & 5 & 68 & 3 & 9 & 2 \\
\hline 24 & Universidad Privada Antonio Guillermo Urrelo & Privada & 5 & 56 & 4 & 2 & 2 \\
\hline 25 & Universidad Católica Los Ángeles de Chimbote & Privada & 5 & 71 & 9 & 2 & 4 \\
\hline 26 & Universidad Católica Santo Toribio de Mogrovejo & Privada & 5 & 72 & 5 & 8 & 2 \\
\hline 27 & Universidad de Chiclayo & Privada & 5 & 49 & 2 & 7 & 1 \\
\hline 28 & Universidad Andina del Cusco & Privada & 6 & 79 & 3 & 2 & 0 \\
\hline 29 & Universidad Nacional De San Antonio Abad del Cusco & Privada & 5 & 72 & 4 & 3 & 1 \\
\hline 30 & Universidad Privada de Huancayo Franklin Roosevelt & Privada & 5 & 59 & 3 & 8 & 0 \\
\hline 31 & Universidad Continental de Ciencias e Ingeniería & Privada & 5 & 58 & 4 & 3 & 0 \\
\hline 32 & Universidad Privada de Huánuco & Privada & 6 & 59 & 5 & 7 & 2 \\
\hline 33 & Universidad Científica del Perú & Privada & 6 & 67 & 4 & 2 & 2 \\
\hline 34 & Universidad Peruana del Oriente & Privada & 6 & 66 & 4 & 2 & 2 \\
\hline 35 & Universidad José Carlos Mariátegui & Privada & 6 & 69 & 5 & 3 & 2 \\
\hline 36 & Universidad Andina Néstor Cáceres Velásquez & Privada & 5 & 57 & 5 & 2 & 0 \\
\hline 37 & Universidad Cesar Vallejo & Privada & 5 & 50 & 4 & 4 & 0 \\
\hline 38 & Universidad Privada de Tacna & Privada & 5 & 65 & 4 & 9 & 2 \\
\hline 39 & Universidad Latinoamericana CIMA & Privada & 6 & 92 & 5 & 2 & 2 \\
\hline
\end{tabular}

* Contabilizado como años de estudio desde el primer semestre hasta el término del internado hospitalario. Dos semestres se contabilizaron como un año de estudios

† Semestre en el cual se imparte la primera asignatura relacionada a investigación científica 


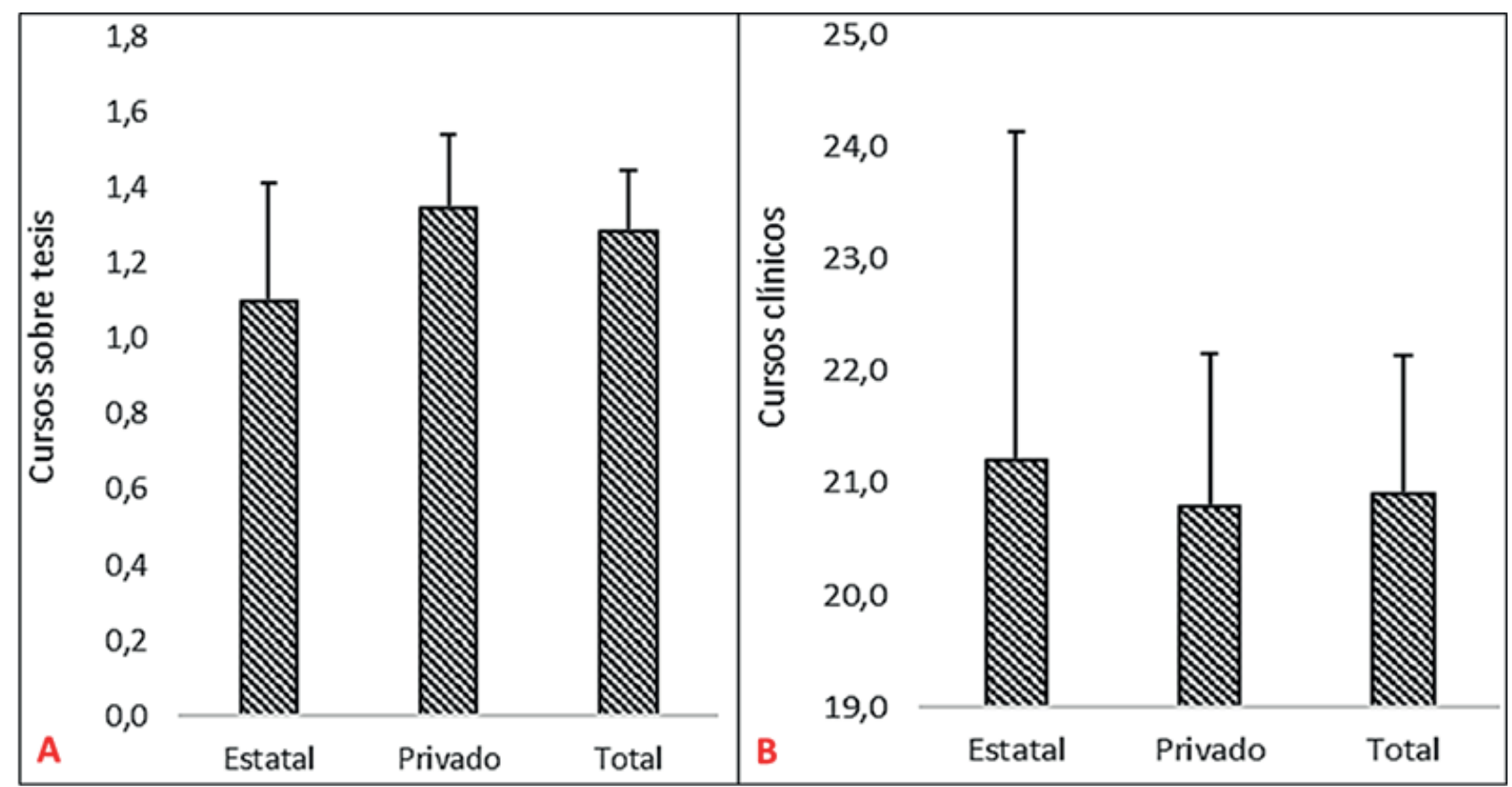

Figura 2. A. Promedio cursos con el término "tesis" por universidad. B. Promedio de cursos clínicos por tipo de universidad

profesional; sin embargo, no se pudo realizar un análisis de la producción científica de cada facultad debido a que en la base de datos Scopus y en los rankings universitarios el área de Odontología ( 'Dentistry') no presenta datos de todas las facultades además de no encontrarse registros de producción científica en los portales web de cada facultad; esto dificulta comparación entre facultades y hace casi imposible analizar si la cantidad de cursos relacionados a la investigación se asocia a una mayor o menor producción de artículos científicos.

A diferencia de esto, en la Medicina Humana sí se cuenta con que dos tercios de las facultades en el Perú presentan artículos en la base de datos Scopus ${ }^{15}$ y quizás presenten docentes como modelos investigadores a seguir ${ }^{16,17}$.

Incluso entre las universidades que publiquen artículos científicos quizás pudiese existir un distanciamiento entre los estudiantes del pregrado y los docentes investigadores o los estudiantes del posgrado (maestrías y doctorados); esto podría enmascarar la producción científica perdiendo la oportunidad de incentivar a un estudiante a que aporte en el desarrollo del conocimiento y quizás perder la oportunidad de seguir la carrera de investigador.

Se encontró que la cantidad de cursos relacionados a la investigación fue heterogénea; si bien no existe un estándar de cuántos y cuáles cursos deberían dictarse para promover la investigación, es recomendable que tales cursos se comiencen a impartir desde los primeros años del pregrado ${ }^{18}$.

Es mejor que los cursos relacionados a la investigación se impartan de forma sucesiva; sin embargo, en la mayoría de veces estos cursos son subestimados por los estudiantes y se enfocan más en los cursos clínicos. Esto refleja el resultado que señala, que el promedio de los cursos clínicos supera ampliamente al promedio de los cursos sobre investigación. Si bien la Odontología requiere que un estudiante adquiera habilidades procedimentales en la atención de los pacientes; no se debe olvidar que la generación del conocimiento y las capacidades investigativas son de igual importancia en el desarrollo profesional.

El 69\% de facultades presentaron cursos relacionados a tesis; hoy en día la SUNEDU (Superintendencia Nacional en Educación) exige que los estudiantes universitarios se titulen bajo una única modalidad: la realización de una tesis ${ }^{19}$. En ese sentido llama la atención que el promedio de cursos sobre tesis sea de 1,3. Si se desea que un estudiante culmine su carrera con la tesis ya terminada se requiere que existan más cursos relacionadas a la tesis, redacción y procesos de sustentación; esto para favorecer que ni bien un estudiante culmine la carrera ya se encuentre apto para sustentar su tesis. Cabe mencionar que en la Odontología peruana el promedio de tiempo que le toma a un estudiante sustentar su tesis, desde que egresa de la facultad, es de dos años ${ }^{20}$.

En el pregrado un estudiante debe seguir etapas para lograr un aprendizaje; en el caso de la investigación, esta debe comenzar con conocimientos básicos, comprenderlos, aplicarlos a través de estudios o aprender de investigadores modelos y finalmente difundirlos a través de informes o artículos; este modelo sigue lo planteado por Miller ${ }^{21}$; sin embargo, en las facultades de Odontología no se aprecia que este modelo llegue a consolidarse; esto se refleja en la nula o escasa producción científica que presenta la Odontología peruana a nivel mundial (según el Scimago al 2017 la PC fue del 0,22\% ${ }^{22}$ ); por lo que quizás las asignaturas solo son teóricas, no llegan a ejecutar proyectos junto con los docentes o no se dictan cursos relacionados a redacción científica. 
Las escuelas profesionales deben realizar sus planes curriculares en base al perfil profesional que requiere la sociedad. Hoy en día la Odontología peruana presenta una superpoblación de odontólogos clínico-asistenciales (principalmente en la ciudad de Lima) y cuyo mayor aprendizaje se basó en el desarrollo de habilidades procedimentales; sin embargo, se adolece de odontólogos que resuelvan problemas de la comunidad, que planteen proyectos, que los difundan y sean partícipes de políticas públicas. Es aquí, donde las habilidades investigativas parten como elementos clave para formar profesionales críticos capaces de solucionar problemas de una sociedad y aumentar la producción científica ${ }^{23}$. Los planes de estudio evaluados en el presente estudio reflejan que, si bien existe un buen número de cursos relacionados a investigación científica en las distintas facultades, cabe preguntarse por qué la producción científica es escaza e incluso nula en la mayoría de ellas. Al parecer no solo es suficiente presentar un plan de estudios apropiado, sino que este se articule con las prácticas docentes apropiadas para lograr las habilidades en los estudiantes. En el caso de la investigación se requiere que los docentes que imparten tales asignaturas demuestren que son capaces de planificar proyectos, ejecutarlos y difundirlos. No es recomendable que un docente que no presenta la experiencia de haber publicado artículos científicos pueda estar enseńando métodos científicos debido a que nunca tuvo la experiencia de culminar un estudio (recordemos que una investigación culmina con la comunicación científica).

Nuestro estudio no está exento de limitaciones y podemos indicar que no se pudo analizar la totalidad de las facultades debido a que tres no presentaron sus planes de estudio en su portal web. Solo nos basamos en el análisis documental de los planes de estudio que no necesariamente reflejan el proceso investigativo de cada facultad. No se pudo analizar el producto final de cada curso de investigación pues esto requiere de un mayor análisis de cada sílabo y los repositorios digitales de cada facultad. Asimismo, la presente investigación debe ser complementada con un estudio de las habilidades investigativas de los estudiantes y relacionarlos con la estructura de sus planes de estudio.

El presente estudio ofrece un marco de referencia descriptivo sobre la estructura de los planes de estudio de las facultades de Odontología del Perú en relación a los cursos sobre investigación científica en el pregrado. Concluimos que el promedio de cursos relacionados a la investigación de las facultades de Odontología en el Perú fue 4,5. Las universidades privadas fueron las que más cursos con el término de "tesis" presentaron en sus planes de estudio con un promedio de $1,3 \pm 0,2$.

\section{Referencias bibliográficas}

1. Laidlaw A, Aiton J, Struthers J, Guild S. Developing research skills in medical students: AMEE Guide No. 69. Med Teach. 2012; 34(9):e754-71. doi: 10.3109/0142159X.2012.704438.
2. Aslam F, Shakir M, Qayyum MA. Why medical students are crucial to the future of research in South Asia. PLoS Med 2005;2:e322.

3. Roldán SL. Elementos para evaluar planes de estudio. Educ. 2005; 29(1):111-23.

4. Neyra M, Berra M, Rodríguez A, Rodríguez R, Reyes G. La estrategia investigativa curricular en la carrera de medicina. Educ Med Super. 1997;11(2):91-100.

5. Huamaní C, Chávez-Solis P, Mayta-Tristán P. Aporte estudiantil en la publicación de artículos científicos en revistas médicas indizadas en Scielo-Perú. An Fac Med (Lima). 2008;69(1):42-5.

6. Castro-Rodríguez Y. Factores que contribuyen en la producción científica estudiantil. El caso de Odontología en la Universidad Nacional Mayor de San Marcos, Perú. Educ Med. 2019;20(S1):49-58. doi: https://doi. org/10.1016/j.edumed.2017.10.002

7. Castro RY, Sihuay-Torres K, Perez-Jimenez V. Producción científica y percepción de la investigación por estudiantes de odontología. Educ Med. 2018;19(1):19-22.

8. Mejia MO, Veramendi-Espinoza L, Huerta-Collado YM, Montenegro-Idrogo JJ. Baja publicación de investigaciones médico estudiantiles curriculares de una universidad peruana. Rev Peru Med Exp Salud Pública. 2014;31(3):608.

9. Pereyra-Elías R, Huaccho-Rojas JJ, Taype-Rondan A, Mejia CR, Mayta-Tristán P. Publicación y factores asociados en docentes universitarios de investigación científica de escuelas de Medicina del Perú. Rev Peru Med Exp Salud Pública. 2014;31:424-30.

10. Molina-Ordóñez J, Huamaní C, Mayta-Tristán P. Apreciación estudiantil sobre la capacitación universitaria en investigación: estudio preliminar. Rev Peru Med Exp Salud Pública. 2008;25:325-9.

11. Ramos-Rodríguez M, Sotomayor R. Realizar o no una tesis: razones de estudiantes de medicina de una universidad pública y factores asociados. Rev Peru Med Exp Salud Publica. 2008;25:322-4.

12. Colegio Odontológico del Perú [Internet]. Directorio de Facultades de Odontología. [Consultado el 21 de enero del 2019]. Disponible en: http://www.cop.org.pe/directorio-de-facultades-de-odontologia.

13. Van Eyk HJ, Hooiveld MH, Van Leeuwen TN, Van der Wurff BL, De Craen AJ, et al. Scientific output of Dutch medical students. Med Teach. 2010;32:231-5.

14. MacDougall M, Riley SC. Initiating undergraduate medical students into communities of research practice: what do supervisors recommend? BMC Med Educ 2010; 10:83. https://doi.org/10.1186/1472-6920-10-83.

15. Taype-Rondán A, Huaccho-Rojas J, Pereyra-Elías R, Mejia CR, Mayta-Tristán P. Características de los cursos de investigación en escuelas de medicina del Perú. Arch Med. 2015 [Consultado el 5 de febrero del 2019]. Accesible en: http://www.archivosdemedicina.com/ medicina-de-familia/caractersticas-de-los-cursos-deinvestigacin-en-escuelas-demedicina-del-per.php?ai$\mathrm{d}=5803 \& \mathrm{fbclid}=\mathrm{IwAR} 2 \mathrm{GG}$ q qWEy0tupW17CopBwpsMizqERLGNtYGFBTDou8ByjzSDTTZKtW62p4. 
16. Levine RB, Lin F, Kern DE, Wright SM, Carrese J. Stories from early-career women physicians who have left academic medicine: a qualitative study at a single institution. Acad Med. 2011;86:752-8.

17. Burgoyne LN, O'Flynn S, Boylan GB Undergraduate medical research: the student perspective. Med Educ Online. 2010; 15. doi: 10.3402/meo.v15i0.5212.

18. Ahmad F, Zehra N, Omair A, Anjum Q. Students' opinion regarding application of epidemiology, biostatistics and survey methodology courses in medical research. J Pak Med Assoc. 2009;59:307-10.

19. Perú, Congreso de la República. Ley 30220: Ley Universitaria. Lima: Congreso de la República; 2014.
20. Castro-Rodríguez Y. Indicadores bibliométricos de las tesis sustentadas por estudiantes de Odontología, Perú. EDUMECENTRO. 2018;10(4):1-19.

21. Miller, G. E. The assessment of clinical skills/competence/ performance. Acad Med. 1990;65:S63-7.

22. Scimago Journal \& Country Rank. [Consultado el 5 de febrero del 2019]. Accesible en: https://www.scimagojr. $\mathrm{com} /$ countrysearch.php? country=pe\&area $=3500$.

23. Pfund C, Maidl-Pribbenow C, Branchaw J, Miller-Lauffer S, Handelsman J. Professional skills. The merits of training mentors. Science. 2006;311:473-4 
